\title{
INFORME DE AUDITORÍA Y SU RELACIÓN CON EL MERCADO
}

INTEGRADO LATINOAMERICANO

(MILA)

The AUdit RePORT AND ITS

RELATIONSHIP WITH THE LATIN

AMERICAN INTEGRATED MARKET

\author{
Belky Esperanza Gutiérrez Castañeda ${ }^{\mathrm{I}}$ \\ Carlos Andrés Barrera Montoya ${ }^{2}$ \\ Daniela Pérez Noreña
}

1 PhD. Universidad de Antioquia. Medellín, Colombia. Correo electrónico: belky. gutierrez@udea.edu.co

Orcid: https://orcid.org/0000-0002-9578-5968

2 Maestría en Economía. Universidad de Antioquia. Correo electrónico: andres. barrera@udea.edu.co

Orcid: https://orcid.org/0000-0002-2235-1843

3 Maestría en Finanzas. Universidad de Antioquia. Correo electrónico: daniela. perezn@udea.edu.co

Orcid: https://orcid.org/0000-0003-0683-6274

Código JeL: G10, G11, G13

Fecha de recepción: 16/07/2019

Fecha de aceptación: 02/09/2019

DOI: https://doi.org/10.18601/16577175.n26.07 


\section{RESUMEN}

El objetivo de la investigación es evaluar la relación del retorno esperado del precio de las acciones de las empresas que cotizan en el mercado integrado latinoamericano (MILA) frente a la divulgación del dictamen del auditor en un periodo 2011-2017, siguiendo la metodología de estudio de eventos (i) Car; (ii) Fama \& French; (iii) BHAR, además test de ARCH, correlaciones tradicionales y cópulas gaussianas. Se identificó que la publicación del dictamen es significativo, sin embargo, no existe una significancia estadísticamente representativa al momento de hacer un modelo de regresión multivariable y de correlación para la relación entre el dictamen y el comportamiento de los precios en los países participantes del miLA.

Palabras clave: dictamen del auditor, mercado integrado latinoamericano (MILA), modelos contabilométricos, estudio de eventos, retornos accionarios, CAR.

\section{Abstract}

The objective of the investigation is to evaluate the relation of the expected return of the stock price of the companies listed in the MILA against the disclosure of the auditor's opinion in a period of 2011-2017, following the methodology of the study of events (i) CAR; (ii) Fama \& French; (iii) BHAR, in addition ARCH test, traditional correlations and Gaussian copulas. It was identified that the publication of the opinion is significant, however, there is no statistically significant significance at the time of making a multivariate regression model and correlation for the relationship between the opinion and the behavior of prices in the participating countries of the MILA.

Key words: Auditor's opinion, Integrated Latin American Market (MILA), accounting models, events study, share returns, CAR.

\section{INTRODUCCIÓN}

El mercado integrado latinoamericano (MILA), durante los últimos años, se ha consolidado como un mercado de valores de gran importancia, ya que reúne en una misma plataforma los valores transados en los mercados de cuatro países, Colombia, México, Perú y Chile, brindando a los inversionistas mayor posibilidades de diversificar sus portafolios de inversión y, a su vez, aumenta la competitividad en el mercado de capitales.

Esto último permite que las empresas muestren una imagen más atractiva de su situación económica y financiera a potenciales inversionistas. En este sentido, el informe del auditor materializado en el dictamen cobra importancia como herramienta a la hora de determinar la fiabilidad de la información consignada en los estados financieros. Por tanto, se presume que este informe podría generar un 
impacto sobre los precios de los activos de los mercados y, en este caso específico, del MILA, por tal motivo el dictamen tiene como principal objetivo aumentar el grado de confianza de los usuarios en los estados financieros.

Así, el dictamen del auditor se convierte en la pieza fundamental de análisis de los inversionistas al tomar decisiones de inversión, por tal motivo este trabajo evalúa la relación del retorno esperado del precio de las acciones de las empresas que cotizan en el MILA frente a la divulgación del dictamen del auditor en un periodo del 2011 al 2017. Mediante la metodología de estudio de eventos, tomando como fecha de evento el día de divulgación del dictamen, se construyeron diferentes modelos de regresión (CAPM, Tres factores de Fama \& French y de volatilidad estocástica), donde la variable dependiente es el retorno anormal acumulado de las empresas y como independientes un conjunto de variables financieras y contables, incluyendo el dictamen del auditor, para así determinar si existen cambios significativos en los retornos esperados de los activos del mercado MILA.

\section{REFERENCIAL TEÓRICO}

\section{Mercado integrado latinoamericano}

Las economías siempre han estado en la búsqueda de la integración de sus mercados, es así que en diferentes contextos económicos han posibilitado el fomento de plataformas regionales de inversión que ha llevado a una expansión de los flujos financieros entre países (Corbo, 1997) un ejemplo de esta integración es el caso de la "integración de las sociedades Meff, Aiaf y Senaf. Se trata de una operación de fusión de tres sociedades rectoras, creando un holding común, para ofrecer un servicio integrado, en las mejores condiciones de calidad y coste" (López Domínguez, 2007, p. 90)

Es así que la integración en Latinoamérica no es la excepción, convirtiéndose esto en una necesidad para aumentar la eficiencia y competitividad frente a otros mercados y así ser una economía más fuerte y consolidada, por esto, en la actualidad, existe el mercado integrado latinoamericano (MILA) que se encuentra constituido por la Bolsa de Valores de Colombia, la Bolsa de Valores de Lima, la Bolsa de Comercio de Santiago y la Bolsa Mexicana de Valores, estas bolsas se unen con el fin de lograr la transacción de activos financieros entre los países miembro, por medio de la integración de sus bolsas de valores y depósitos centrales de valores.

Igualmente, el MILA brinda a los inversionistas la posibilidad de diversificar sus carteras por medio de la adquisición de títulos valores de las empresas enlistadas en las bolsas de valores de cualquiera de los países miembro, así los inversionistas pueden obtener activos sujetos a condiciones diferentes a las de aquellos que se transan en la misma zona geográfica en que estos residen, además de "lograr un mercado único latinoamericano que les brinde a inversionistas nacionales e internacionales mayores oportunidades de diversificación de sus portafolios y satisfaga sus necesidades de inversión y, de igual forma, otorgue beneficios a los emisores 
internacionales" (Romero, Ramírez, y Guzmán, 2013, p. 55). Dicho mercado también brinda a los inversionistas el mejoramiento del balance riesgo-retorno y la disposición de más instrumentos financieros para diversas operaciones de compra y venta, mientras que para los emisores, el acceso a nuevos mercados y un aumento en la demanda de los activos emitidos, adicional de permitir la complementación de los mercados, al unir industrias y sectores económicos (Vargas y Bayardo, 2013).

Ahora bien, en la medida que el MiLA cobra importancia como un mercado accionario que busca fortalecer los mercados de los países que pertenecen a esta integración, los inversionistas constantemente buscan herramientas para la toma de decisiones que sean confiables, tales como análisis de los estados financieros, calificaciones de riesgo país, opiniones de expertos, análisis técnicos y fundamentales del comportamiento de las empresas, evaluaciones macroeconómicas, que ayuden a visualizar situaciones políticas, económicas y sociales en los países que se desea invertir. Sin embargo, existe otra herramienta, además de las ya mencionadas, como es el informe del auditor que brinda una opinión de que los estados financieros de las organizaciones son fiel reflejo de las empresas, brindada esta por una persona externa e independiente para brindar confiabilidad y seguridad a los inversionistas, esta última herramienta se tomara como referente para el análisis de la relación existente entre el dictamen de auditoria y los precios de los activos del miLA.

\section{Auditoria y mercados accionarios}

La auditoría se puede definir como un examen, revisión, evaluación independiente y objetiva de hechos, procedimientos, documentos y demostraciones contables, con el fin de verificar y comprobar la razonabilidad y el cumplimiento de lo determinado por la administración, la ley, el Estado y la sociedad, para emitir un informe u opinión (Franco y Marra, 2000), dicho informe de auditoría desempeña un papel importante en las economías de mercado y, en particular, en los mercados accionarios, ya que actúa como fuente de información a través del análisis y divulgación del dictamen del auditor de las compañías; sin embargo, para que este sea de utilidad para los inversores, es necesario que sea fidedigna con la realidad económica de las empresas, es así como la auditoría se concibe como un proceso base para la emisión del dictamen, ya que busca expresar una opinión sobre determinado hecho económico, administrativo y contable de una empresa, pudiendo esto afectar los precios de las acciones, debido a que este puede contener información que afecte a la estimación de los flujos de efectivo futuros y el grado de riesgo de estos flujos, también porque puede contener información sustancial sobre la viabilidad de la empresa; por ejemplo, opiniones sobre su continuidad operativa, por esto es que se espera que este informe de auditoría afecte la apreciación de los inversores sobre el desempeño normal de una compañía en los mercados accionarios, ya que al ser la principal función de la auditoría dar confianza sobre las transacciones de mercado a través de la emisión de una opinión i) con salvedad; (ii) sin reserva; (iii) 
adverso o (iv) abstención de opinión, sobre los estados financieros que permiten a los inversionistas tener información relevante de las organizaciones (Dye, 1993).

Es así como la opinión del auditor ha sido considerada de gran importancia para mantener el grado de confianza y seguridad en una economía, debido a que, como lo expresa Wallace (1987), la auditoria desempeña un papel importante, ya que cumple funciones de monitores, de información y seguridad, esto en la medida en que permite ejecutar acciones oportunas para anticiparse a problemas, garantizar sostenibilidad de los procesos y retroalimentar estos en las organizaciones a través del monitoreo. Además, la información en los procesos de auditoria cumple un papel fundamental en la medida en que i) da herramientas para la reducción del riesgo, (ii) mejora la toma de decisiones y (iii) mejora los beneficios obtenidos en las negociaciones en un mercado accionario (Fama y Laffer, 1971). Ahora bien, el informe de auditoría no solo brinda herramientas para la toma de decisiones de los inversionistas, sino que también ayuda en las organizaciones a la mejora de la calidad de los datos utilizados en la presentación de estados financieros, en la toma de decisiones internas de las empresas, ayuda a detectar errores y a obligar a los empleados a ser más cuidadosos en la elaboración de los registros contables, porque a medida que los inversores evalúan la información contable para tomar decisiones de inversión al no tener seguridad para invertir sus excedentes de capital, estos exigen un mayor retorno a los niveles más altos de riesgo.

Por lo anterior, durante varios años, muchos autores han direccionado sus esfuerzos a identificar la relación del dictamen de auditoria con el impacto de este en los mercados accionarios, ya que este puede generar variaciones en los precios de las acciones.

Dopuch, Houlthausen y Leftwich (1986) analizaron el efecto de los precios de las acciones en relación con la divulgación de los informes de auditoría a través de los medios de comunicación, tuvo un efecto diferente de aquellos divulgados, utilizando solo el formulario $10-\mathrm{K}$, obteniendo como resultado significativamente negativo del precio de las acciones ante la divulgación de los informes de auditoría con salvedad del tipo "sujeto a”. Por otro lado, Soltani (2000) analizó 543 informes de auditoría, en el periodo 1986-1995, en el mercado de capitales francés, calculando el efecto del evento de divulgación a través de varios modelos de por tres ventanas de evento, obteniendo con lo anterior que los diferentes tipos de informes de auditoría con salvedad tienen significativos retornos anormales negativos alrededor de las fechas de su anuncio, además, se presentaron retornos anormales significativos para las compañías que recibieron informes de auditoría con excepción del tipo "observations" y "remarks", indicando que los usuarios de la información de auditoría pueden estar principalmente interesados en el contenido del informe de auditoría en su totalidad, más que en el contenido de un solo y específico párrafo del informe.

Por otro lado, Martínez et al. (2004) encontraron que los informes de auditoría no proporcionan al inversor contenido informativo y que el impacto fue insignificante, dependiendo de si los informes fueron emitidos por grandes o pequeñas 
empresas de auditoría, esto debido a que en ocasiones los inversionistas cuentan con información anticipada o hay rumores en el mercado de que las compañías probablemente recibirían salvedad, mucho antes de que los informes fueran publicados oficialmente. Por otra parte, Chen et al. (1996) estudió el impacto de los informes de auditoría en el precio de las acciones de empresas listadas en la bolsa de Shanghai en el periodo 1995-1997, teniendo como resultado que i) los informes de auditoría con salvedad se asociaron con significativos retornos negativos de mercado, ii) no se encontró ninguna diferencia entre el impacto de las opiniones de auditoría elaboradas según los GAAP y iii) no encontraron ninguna reacción significativa del mercado en relación con los informes sin salvedad, pero con notas explicativas. Pei, Opong y Hamill (2004) evaluaron el contenido de los informes de auditoría de las compañías enumeradas en la bolsa de Shenzhen, encontrando que el mercado reacciona a los informes con salvedad; sin embargo, el impacto fue significativo dos días antes de su publicación oficial, mostrando que el mercado descontó el efecto del evento antes de su divulgación.

\section{Metodología}

La investigación se desarrolla bajo un enfoque cuantitativo, de alcance correlacional, utilizando la metodología de Fu (2009), donde es necesario que cada acción cotice un mínimo de quince días durante cada mes del periodo de la muestra. El conjunto de empresas analizadas fueron las pertenecientes al mercado integrado latinoamericano mILA, en los cuales se evaluaron los dictámenes emitidos por el auditor a los estados financieros del 2010 al 2017.

El proceso de selección de la muestra comenzó considerando solo las acciones ordinarias que estaban presentes en los meses del periodo de estudio, adicionalmente, siguiendo los criterios de Fama y French (1993) se excluyen las acciones de bancos, seguros y fondos de inversión, empresas con acciones preferenciales y las que reportaron el 31 de diciembre un patrimonio negativo (Mendonça, Klotzle, Pinto y Montezano, 2012). Con el fin de identificar la existencia de cambios significativos en los precios de las acciones del mila se usó la metodología de estudio de evento de Fama (1991) y Mackinlay (1997), donde se define como fecha de evento la fecha de divulgación del dictamen de auditoria de los estados financieros del 2010 al 2017 y considerando ventanas de evento los intervalos de tiempo $(-2,2),(-5,5)$ y $(-10,10)$ y una ventana de estimación sesenta días hábiles previos a la ventana de evento (gráfico 1).

Ahora bien, con el objetivo de identificar los cambios en los retornos esperados derivados de la divulgación del dictamen de auditoria se calculó el retorno anormal acumulado de las acciones durante el periodo de estudio bajo tres modelos existentes en la literatura: modelo de mercado, modelo de tres factores de Fama y French y rendimiento anormal (внаR). Ahora, con el fin verificar el objetivo de la investigación, es necesario definir y construir un conjunto de variables financieras utilizadas en la relación existente entre el dictamen del auditor y los retornos 
esperados, determinando en las ecuaciones descritas, a continuación, los modelos para estimar la variable dependiente desde varios enfoques.

Gráfico 1. Esquema estudio de evento y ventana de estimación

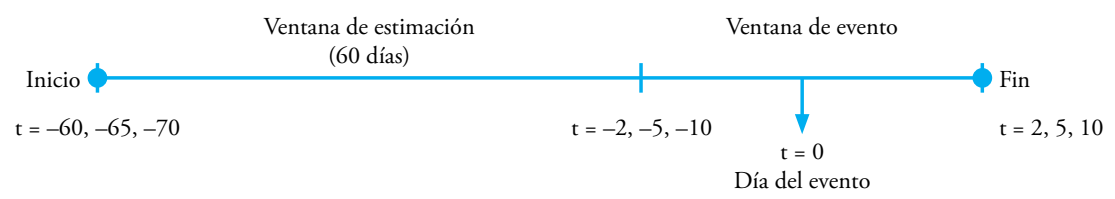

Fuente: elaboración propia.

De acuerdo con lo anterior, en el estudio se calcularon los retornos anormales acumulados en principio bajo el modelo de mercado donde se compara el retorno real con los retornos estimados, para así obtener el retorno anormal (ver ecuación 1).

$$
A R_{i t}=R_{i t}-E\left[R_{i t} / X\right]
$$

Donde $A R_{\text {itevento }}$ representa el retorno anormal de la acción i en la fecha del evento, $\widetilde{R}_{i, t_{\text {evento }}}$ el retorno real de la acción i en la fecha del evento y $E\left[R_{i t} / X_{t}\right]$ muestra el retorno esperado de la acción i en la fecha t evento por el retorno $X_{\text {tevento }}$, este último se denota:

$$
A R_{i, t_{\text {evento }}}=\widetilde{R}_{i, t_{\text {tenuto }}}-R_{\text {normal }_{i, \text { equno }}}
$$

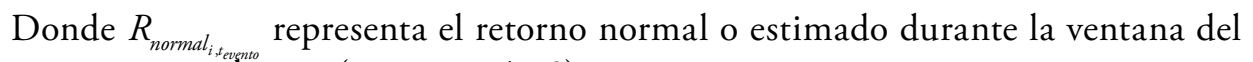
evento que se denota (ver ecuación 3).

$$
\begin{aligned}
R_{\text {normal }}=\alpha_{i}+\hat{\beta}_{i} \check{R}_{i t}+\hat{\varepsilon}_{i, t} \\
\check{R}_{i t}=\ln \left(\frac{P_{i, t}}{P_{i, t-1}}\right)
\end{aligned}
$$

Donde, $\check{R}_{i t}$ es el rendimiento de las acciones del mercado mila en el periodo $t$ tranformado por logaritmo natural entre el precio de la acción i en el periodo $t$ y $t-1, \alpha_{i}$ el intercepto, $\hat{\beta}_{i}$ el coeficiente de la pendiente, $\hat{\boldsymbol{\varepsilon}}_{i, t}$ el error en el proceso de generar rentabilidad en el periodo $t$.

Ahora bien, para el cálculo del retorno anormal promedio por el modelo de mercado se tiene la siguiente expresión (ver ecuación 4). 


$$
C A R_{i}=\sum_{t_{1}}^{t_{2.510}} A R_{i, t_{\text {centolo }}}
$$

Donde $\mathrm{CAR}_{i}$ representa el retorno anormal acumulado de la empresa $i, t_{1}$ primer día del evento y $t_{2}$ último día del evento.

Por otro lado, para el cálculo de los retornos anormales acumulados por el modelo de Fama y French (1993) y siguiendo la metodología de Fu (2009) donde se explican los retornos de los activos en función del exceso de retorno del mercado, el tamaño de las empresas sмв (small minus big), el retorno del portafolio en función del factor alto Book to Market (HML) (high minus low) (Nieto, 2001) (ver ecuación 5).

$$
R_{i \tau}-r_{\tau}=a_{i t}+b_{i t}\left(R_{m \tau}-r_{t}\right)+S_{i t} S M B+h_{i t} H M L+\varepsilon_{i t}
$$

Donde $\tau$ es el día búrsatil, $t$ indica el mes, $R_{i \tau}$ es el retorno de acción i, $r_{t}$ es la tasa de interés libre de riesgo (tasa del tesoro de estados unidos de diez años), $R_{m \tau}$ es el retorno de mercado (índices de mercado S\&P mILA Andean 40), $b_{i t}, S_{i t}$ e $h_{i t}^{m \tau}$ son de cada uno de los tres coeficientes de los factores de Fama y French y $\varepsilon_{i t}$ son los errores del modelo los cuales distribuyen de forma normal con media cero y varianza no constante $\varepsilon_{i t} \sim N\left(0, \sigma_{i, t}^{2}\right)$. Ahora, el cAR de Fama y French se calcula por medio de la ecuación 6 definida por

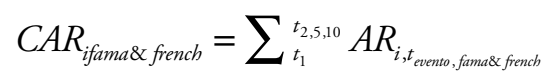

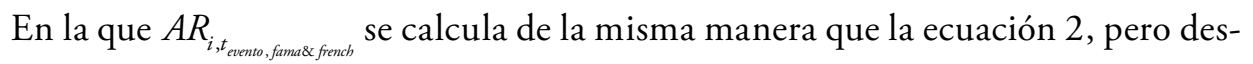
contando la tasa libre de riesgo a cada retorno para la estimación de los coeficientes $\alpha_{i}$ y $\beta_{i}$ del modelo de mercado y el de Fama y French se calculó a través de test de ARCH, ya que según Bollerslev (1986), Akgiray (1989) y Chiang y Doong (2001) los retornos de las acciones presentan volatilidad variable a lo largo del tiempo, debido a que tienden a mostrar efectos ARCH (autorregresivo condicionalmente heteroscedástico), lo anterior se hace en principio estimando el modelo por oLs, pero al este no tener en cuenta los residuos se estima nuevamente el modelo por ARCH o GARCH (heteroscedastic autorregresivo generalizado) (ver ecuación 7):

$$
\begin{gathered}
R=R_{m}+u \quad u_{i t} \sim N\left(0, \sigma_{i t}^{2}\right) \\
\sigma_{i t}^{2}=\alpha_{i t}+\alpha_{i 1} u_{i t}^{2}+\alpha_{i 2} \sigma_{i t-1}^{2}
\end{gathered}
$$


Finalmente, se estimó el rendimiento anormal (BHAR) que se fundamenta en los rendimientos compuestos (Buy and Hold Returns), donde se calculan los rendimientos anormales a largo plazo a partir de los rendimientos a corto plazo para obtener el rendimiento en la ventana del evento, siguiendo la estrategia de comprar y mantener durante dicho periodo (Barber y Lyon, 1997). En inicio se estima el rendimiento de la ventana de evento para un título i dado por la expresión:

$$
B H R_{i t}=\left[\prod_{t=s}^{s+t}\left(1+R_{i t}\right)\right]
$$

Donde $R_{i t}$ es el rendimiento de la empresa i de la muestra en el mes $t$.

Dicha rentabilidad calculada $\left(B H R_{i t}\right)$ para las empresas de la muestra se compara con un rendimiento de referencia, para el caso de la investigación con el índice del mercado mILA S\&P mILA Andean 40, para así obtener una rentabilidad anormal a partir del rendimiento de un activo y el rendimiento esperado de este en la ventana de evento:

$$
B H A R_{i t}=B H R_{i t}-B H R_{c o n t r o l, t}
$$

Donde $B H R_{\text {control, }}$ es definido a partir de los rendimientos del mercado.

De acuerdo con los modelos descritos anteriormente y con el fin de determinar la significancia de los retornos anormales acumulados por los tres modelos se calculó el estadístico T-Student, con $N-2$ grados de libertad y una probabilidad en nivel de significancia $\alpha=0.05$ (ver tabla 1 ).

\begin{tabular}{|c|c|c|}
\hline Modelo de mercado & Fama y French & Rendimiento anormal (BHAR) \\
\hline$t=\frac{\overline{C A R}}{\frac{\sigma A R}{\sqrt{n}}} \sim t_{N-2}$ & $t=\frac{\overline{C A R F \& F}}{\frac{\sigma A R F \& F}{\sqrt{n}}} \sim t_{N-2}$ & $t=\frac{B H A R_{t}}{\frac{\sigma B H R}{\sqrt{n}}} \sim t_{N-2}$ \\
\hline $\begin{array}{l}\overline{C A R}: \text { es el promedio de los } \\
\text { rendimientos anormales. } \\
\sigma A R: \text { es la desviación de los } \\
\text { retornos anormales. } \\
n: \text { es el número de evento. }\end{array}$ & $\begin{array}{l}\overline{C A R F \& F} \text { : es el promedio de los } \\
\text { rendimientos anormales calculados } \\
\text { por el modelo de Fama y French. } \\
\sigma A R F \& F: \text { es la desviación de los } \\
\text { retornos anormales por el modelo } \\
\text { Fama y French. } \\
n \text { : es el número de evento. }\end{array}$ & $\begin{array}{l}B H A R_{t} \text { : es el promedio de los } \\
\text { rendimientos anormales. } \\
\sigma B H R \text { : es la desviación de la } \\
\text { rentabilidad promedio. } \\
n \text { : es el número de evento. }\end{array}$ \\
\hline
\end{tabular}

Tabla 1. Estadísticos T-Student retornos anormales acumulados 
Si bien es de suma relevancia establecer para la investigación el impacto del dictamen del auditor en los retornos esperados de los activos del mercado miLA y hacer una prueba de validación de los resultados, es relevante plantear un modelo en el que se evalue si dicho impacto es solo por el dictamen y si existe la posibilidad de que los retornos anormales de las empresas se relacionen con otras variables, las cuales pueden ser significativas a la hora de explicar su comportamiento, por tal motivo se plantea un modelo de regresión de corte transversal (ver ecuacion 10).

$$
\begin{gathered}
\text { CAR }_{t}^{*}=\beta_{1}+\beta_{2 t}{\text { Dummy } \text { Dictamen }_{i t}+\sum_{K=2}^{K} \beta_{k t} X_{k t}+\varepsilon_{t}}^{t=1,2, \ldots, 278}
\end{gathered}
$$

Donde $C A R_{t}^{*}$ es el retorno anormal calculado por cada una de las metodologías mencionadas anteriormente, $X_{k i t}$, representan otras variables como

Tabla 2.

\begin{tabular}{|l|l|}
\hline Dummy Dictamen & $\begin{array}{l}\text { Variable dummy que toma valores de (1) cuando el dictamen emitido recibió } \\
\text { calificación sin salvedad o limpio y (0) en caso contrario. }\end{array}$ \\
\hline LnAct & $\begin{array}{l}\text { Logaritmo natural del total de activos, entendido como el tamańo de la } \\
\text { empresa. }\end{array}$ \\
\hline UPA & $\begin{array}{l}\text { Utilidad por acciones, representa la división del total de la utilidad anual neta } \\
\text { sobre el número de acciones en circulación. }\end{array}$ \\
\hline ROA & $\begin{array}{l}\text { Rentabilidad del activo, relación entre la utilidad de un periodo y los activos } \\
\text { totales. }\end{array}$ \\
\hline ROE & $\begin{array}{l}\text { Rentabilidad del patrimonio, rendimiento que obtienen los accionistas de } \\
\text { una empresa. }\end{array}$ \\
\hline Dummy Ganancia & $\begin{array}{l}\text { Variable dummy de la utilidad neta toma valores de (1) cuando la empresa de } \\
\text { la muestra tiene utilidad positiva o (0) en caso contrario. }\end{array}$ \\
\hline
\end{tabular}

Fuente: elaboración propia.

Ahora, con el conjunto de variables independientes de estudio ya definidas, el siguiente paso consistió en el análisis correlacional entre las variables, por tanto, en el estudio se utilizó las cópulas multivariantes que son las más aplicables en la práctica para modelar la dependencia entre variables financieras cuando la correlación lineal no es suficiente (correlación asimétrica de activos), la cual no puede ser percibida por el coeficiente de correlación de Sperman, la cual es la medida de dependencia más popular en finanzas. Existen diferentes tipos de cópulas en la literatura, como la cópula Gaussiana (o normal), la cópula t-student, la cópula arquimedianas, entre otras (Cardona, 2012; Mendoza y Galvanovskis, 2014). Al 
igual que en el caso de la correlación de Sperman, este tipo de cópulas arroja un coeficiente de correlación el $\rho \in[-1.1]$. El análisis de los resultados de $\rho$ son semejantes al de Sperman, para los valores cercanos a -1 y 1 se tienen correlaciones negativas o positivas, respectivamente, cero, significa no correlación, pero no independencia. En el gráfico 2, se puede observar el gráfico de una cópula gaussiana.

\section{Gráfico 2. Cópula gausiana}

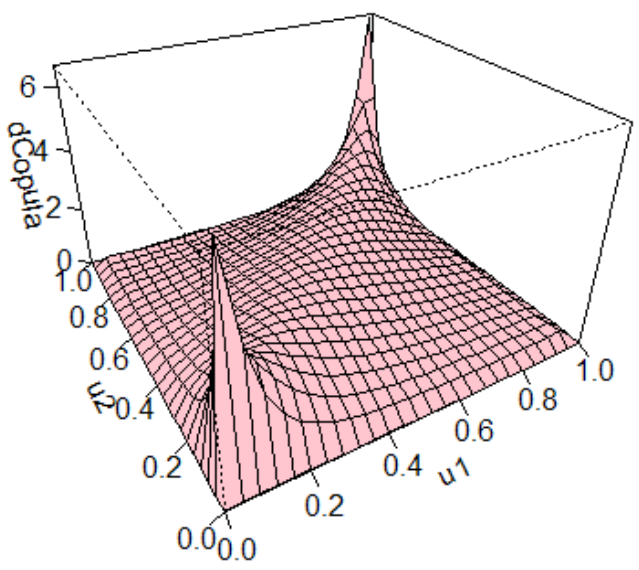

Fuente: elaboración propia.

\section{Resultados Y DISCUSIÓN}

En la tabla 3 se ilustran los resultados encontrados para los retornos anormales esperados, estimados por los diferentes modelos, además en la tabla 4 se ilustran las estadísticas descriptivas del estadístico de prueba para cada una de las variables dependientes del estudio.

Tabla 3. Estadísticas descriptivas promedio

de retornos anormales acumulados MILA

\begin{tabular}{|l|c|c|c|c|c|c|c|c|c|}
\hline & CAR2 & CAR5 & CAR10 & CARF\&F2 & CARF\&F5 & CARF\&F10 & BHAR2 & BHAR5 & BHAR10 \\
\hline Media $\bar{X}$ & .0013 & .0086 & 0.0173 & .2439 & .2637 & .2794 & .0026 & .0074 & .0093 \\
\hline Mediana $M_{d}$ & .0027 & .0020 & .0091 & -.0628 & -.0707 & -.8083 & .0027 & .0055 & .0057 \\
\hline Desv. típ. $\sigma$ & .0668 & .0856 & .1327 & 6.6066 & 10.3386 & 12.8856 & .0510 & .0689 & .1020 \\
\hline
\end{tabular}

Fuente: elaboración propia. 
Los resultados muestran que en general los retornos anormales de las empresas son positivos por cada uno de los tres modelos estimados y para cada una de las ventanas de eventos utilizadas, indicando que el mercado accionario ante eventos, como los dictámenes de auditoria, los retornos promedio de las acciones son positivas.

Tabla 4. estadísticos T-Student promedio y porcentaje de empresas significativas

\begin{tabular}{|l|c|c|c|c|c|c|c|c|c|}
\hline & CAR2 & CAR5 & CAR10 & CARF\&F2 & CARF\&F5 & CARF\&F10 & BHAR2 & BHAR5 & BHAR10 \\
\hline T-Student $\bar{T}$ & 5.7899 & 11.0994 & 21.5691 & 5.8574 & 12.4844 & 24.1924 & 5.6219 & 9.6305 & 17.6811 \\
\hline $\begin{array}{l}\text { Porcentaje } \\
\text { significancia }\end{array}$ & $58.27 \%$ & $84.53 \%$ & $91.01 \%$ & $57.19 \%$ & $87.05 \%$ & $91.73 \%$ & $62.23 \%$ & $79.14 \%$ & $86.33 \%$ \\
\hline
\end{tabular}

Fuente: elaboración propia.

Adicionalmente, en la tabla 4 se ilustra el promedio de los estadísticos $t$ el porcentaje de empresas con un estadístico $|t(N-2)| \geq 3.1824$, al nivel de significancia $\alpha=$ 0.05 . Se puede verificar que este promedio mejora al aumentar con el tamańo de la ventana de evento sin importar el modelo de estimación utilizado para calcular el CAR.

En el gráfico 3, se ilustra la evolución en series de tiempo para cada uno de los CAR estimados en la investigación, en general, estas tienen un comportamiento homogéneo en el tiempo, indicando que las metodologías utilizadas para estos cálculos no difieren, a excepción del modelo por Fama y French, debido a que este toma en cuenta variables adicionales como lo el tamaño y la capitalización de las empresas objeto de estudio, además de descontar los retornos de los activos y de mercado a la tasa libre de riesgo.

Gráfico 3. Series de retornos anormales acumulados MILA

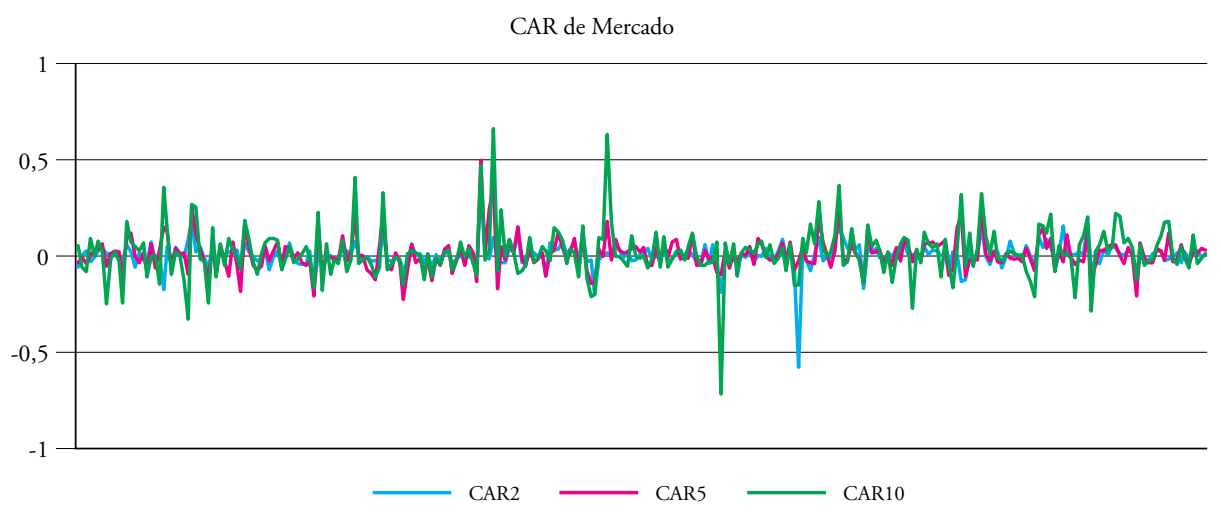


CAR Fama \&French

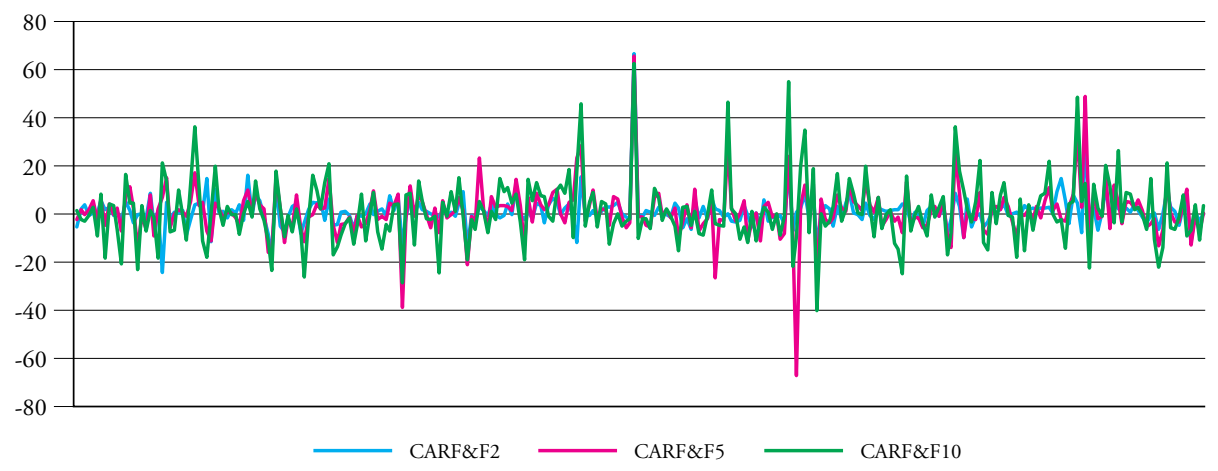

Rendimiento anormal (BHAR)

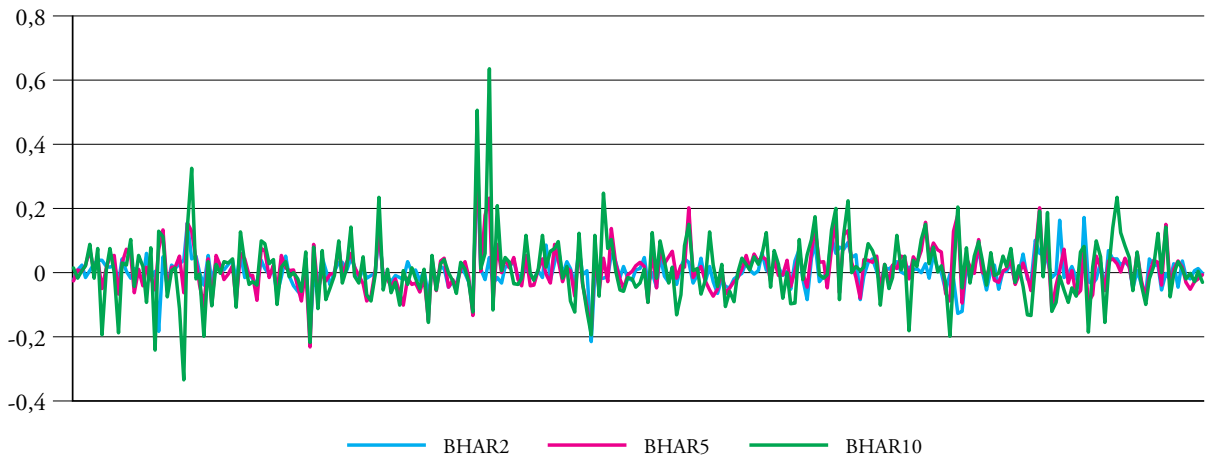

Fuente: elaboración propia.

En la tabla 5 se muestra la estadística descriptiva promedio de cada una de las variables independientes usadas en el modelo de regresión y en la tabla 6 se exponen las correlaciones promedio entre cada una de las variables independientes para así verificar cuál relación hay entre ellas con los retornos anormales acumulados calculados por el modelo de mercado, Fama y French y BHAR en las diferentes ventanas de eventos planteadas.

Tabla 5. Estadísticas descriptivas de las variables independientes del modelo

\begin{tabular}{|l|c|c|c|c|c|c|}
\hline & Dummy Dictamen & LnAct & UPA & ROA & ROE & Dummy Ganancia \\
\hline Media $\bar{X}$ & .9856 & 14.8383 & .4106 & 4.3728 & 8.1126 & .8921 \\
\hline Mediana $M_{d}$ & 1.0000 & 14.7832 & .1124 & 4.0733 & 8.9999 & 1.0000 \\
\hline Desv. típ. $\sigma$ & .1193 & 1.4520 & 1.4698 & 6.4318 & 24.0356 & .3108 \\
\hline
\end{tabular}

Fuente: elaboración propia. 
La correlación de Sperman para el mercado MILA entre los retornos anormales en las diferentes ventanas de eventos es positiva y significativa a nivel de $1 \%$, como lo ilustra la tabla 6 , indicando que los tres tipos de car calculados en el estudio sirve para mostrar el comportamiento de las empresas de este mercado ante eventos, como el dictamen de los auditores. Adicionalmente, se puede visualizar en esta tabla que solo existe correlación significativa entre el CARF\&F2 y el dictamen del auditor, lo cual permite concluir que, en general, los dictámenes de auditoria no generan retornos anormales en este mercado durante el periodo 2011 al 2017.

En la tabla 7 se presentan los resultados de las regresiones entre los retornos anormales acumulados en las ventanas de evento y otras variables que según la teoría financiera pueden influir en los retornos anormales. Inicialmente, se estimó un modelo de regresión lineal en el que la variable dependiente es el CAR y las independientes son variables como ROE, ROA, UPA, ganancia-perdida, posteriormente a cada modelo se le realizó test de heterocedastidad de ARCH (autorregresivo condicionalmente heteroscedástico). En cada uno de los modelos se estimaron los coeficientes beta de cada variable, así como su significancia individual, el Rcuadrado y la significancia global por modelo ols y ARCH. En general, se puede observar que ninguna variable independiente es significativa implicando así que ningún modelo estimado tiene buen ajuste a nivel de significancia del $5 \%$, esto último permite afirmar que dichos retornos anormales no se presentaron por características propias de las empresas.

En relación con las correlaciones presentadas en la tabla 6 y los modelos de regresión de la tabla 7 , se realizó una prueba robustez por medio de las cópulas gaussianas (ver tabla 8), en las que se hizo nuevamente el cálculo de correlación entre las variables CAR para cada una de las ventanas de eventos y la variable que representa dictamen de auditoria en el mercado integrado mila. En este sentido, las correlaciones mejoran a un nivel de significancia del $5 \%$ para todas las incluidas en este análisis comparado con la tabla (correlación Pearson), pero para el caso de la correlación de DummyDictamen con cada uno de los retornos anormales calculados, solo se encuentra significancia con las variables CAR2, CARF\&F2 y BHAR2, dado que, en este caso, el Rho es mayor a 0.2. Los resultados muestran que las cópulas perciben mejor la correlación entre las variables financieras analizadas, pero según lo encontrado en las tablas 6 y 7 la variable dictamen no es capaz de ajustar los retornos anormales del conjunto de empresas utilizadas de este mercado integrado analizadas en el estudio. 


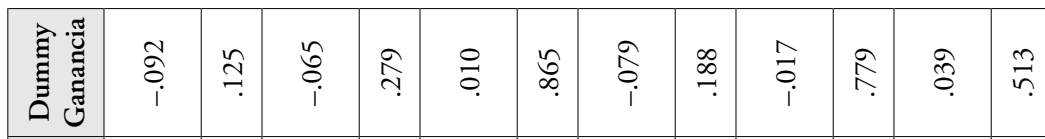

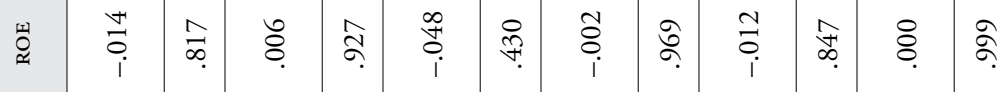

\begin{tabular}{|c|c|c|c|c|c|c|c|c|c|c|c|}
\hline$\underset{\mathscr{\Omega}}{\overleftarrow{a}}$ & $g$ & $\stackrel{\Re}{\sim}$ & $\dot{b}_{i}$ & $\begin{array}{l}\text { ?ี } \\
\text { ?. }\end{array}$ & $\stackrel{\infty}{o}$ & 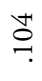 & $\ddot{g}$ & $\approx$ & ఓֶ. & $\frac{+}{\hat{\sigma}}$ & के \\
\hline
\end{tabular}

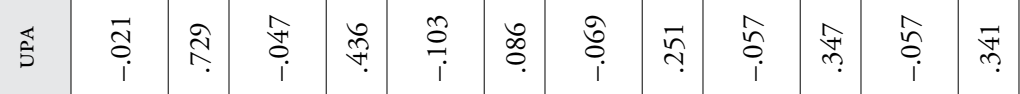

\begin{tabular}{|c|c|c|c|c|c|c|c|c|c|c|c|}
\hline 节 & $\underset{i}{\stackrel{*}{0}}$ & $\stackrel{\overbrace{}}{\stackrel{\Upsilon}{~}}$ & $\ddot{\delta}$ & $\underset{\sim}{\sim}$ & $\tilde{\delta}$ & $\stackrel{\infty}{\stackrel{\infty}{\Upsilon}}$ & $\widehat{\widehat{o}}$ & $\sqrt{t}$ & $\underset{\hat{\sigma}}{\widehat{a}}$ & $\infty$ & $\stackrel{0}{\circ}$ \\
\hline 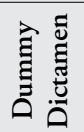 & 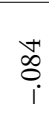 & $\underset{-}{\tilde{0}}$ & $\stackrel{0}{0}$ & & $\stackrel{\infty}{\stackrel{\infty}{0}}$ & $\stackrel{\mathfrak{o}}{\uparrow}$ & $\stackrel{3}{1}$ & సิ & fo & $\underset{乛}{\nabla}$ & $\underset{i}{0}$ \\
\hline
\end{tabular}

\begin{tabular}{|c|c|c|c|c|c|c|c|c|c|c|c|c|}
\hline $\begin{array}{l}\text { 蛋 } \\
\text { 壳 }\end{array}$ & $\begin{array}{l}* \\
\text { ஸे }\end{array}$ & \& & $\begin{array}{l}\text { t+t } \\
n \\
n\end{array}$ & \& & $\begin{array}{l}\text { * } \\
\stackrel{0}{1}\end{array}$ & 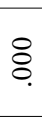 & ֶ̂. & 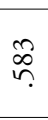 & $\stackrel{*}{\curvearrowright}$ & $\overline{8}$ & సิ & 8 \\
\hline 命 & $\stackrel{*}{\stackrel{*}{\sigma}}$ & 8 & 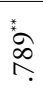 & 8 & రి & 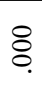 & 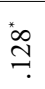 & ชิ & $\stackrel{*}{\stackrel{*}{N}}$ & 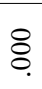 & ำ & $\overline{8}$ \\
\hline
\end{tabular}

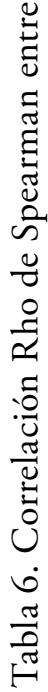

\begin{tabular}{|c|c|c|c|c|c|c|c|c|c|c|c|c|}
\hline ્ָર & $\begin{array}{l}\text { *0 } \\
\text { న్ }\end{array}$ & 8 & $\stackrel{*}{\stackrel{*}{*}}$ & 8 & $\underset{\sim}{\sim}$ & 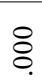 & ㅊ. & $\stackrel{+}{\circ}$ & $\stackrel{\star}{\widehat{\sigma}}$ & สิ & $\stackrel{\circ}{\circ}$ & $\tilde{\sim}$ \\
\hline
\end{tabular}

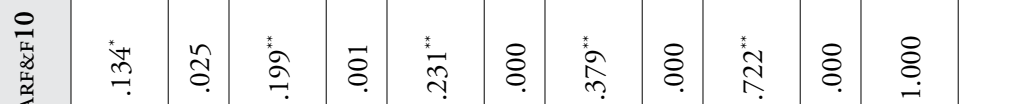

\begin{tabular}{|c|c|c|c|c|c|c|c|c|c|c|c|c|}
\hline 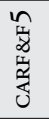 & $\begin{array}{l}* \\
\text { ते } \\
\text { त. }\end{array}$ & $\stackrel{8}{\circ}$ & $\stackrel{*}{\stackrel{*}{\vartheta}}$ & \&. & $\stackrel{*}{\stackrel{*}{\sim}}$ & $\stackrel{8}{0}$ & 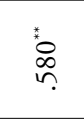 & \&̊ & $\stackrel{8}{8}$ & & & \\
\hline 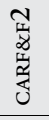 & $\stackrel{*}{\stackrel{*}{ヘ}}$ & $\stackrel{8}{8}$ & $\underset{\sim}{*}$ & $\hat{\tilde{o}}$ & $\hat{n}$ & $\begin{array}{l}\text { T్ } \\
\text { nి }\end{array}$ & $\stackrel{8}{\&}$ & & & & & \\
\hline 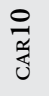 & के & \& & t: & \& & ¿̊. & & & & & & & \\
\hline ఇ్ֶ & 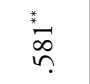 & \& & ¿̊. & & & & & & & & & \\
\hline 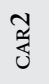 & ¿̨. & & & & & & & & & & & \\
\hline & 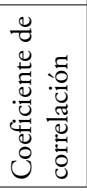 & 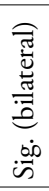 & 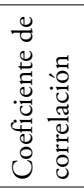 & 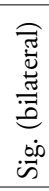 & 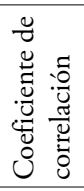 & 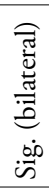 & 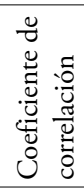 & 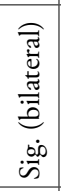 & 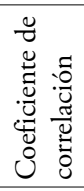 & 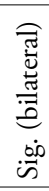 & 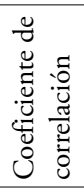 & 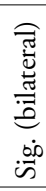 \\
\hline & \multicolumn{2}{|c|}{ 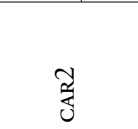 } & \multicolumn{2}{|c|}{ ఇ్ֶ } & \multicolumn{2}{|c|}{ 을 } & \multicolumn{2}{|c|}{ 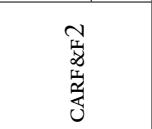 } & \multicolumn{2}{|c|}{ 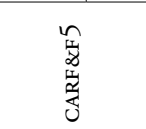 } & \multicolumn{2}{|c|}{ 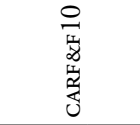 } \\
\hline
\end{tabular}




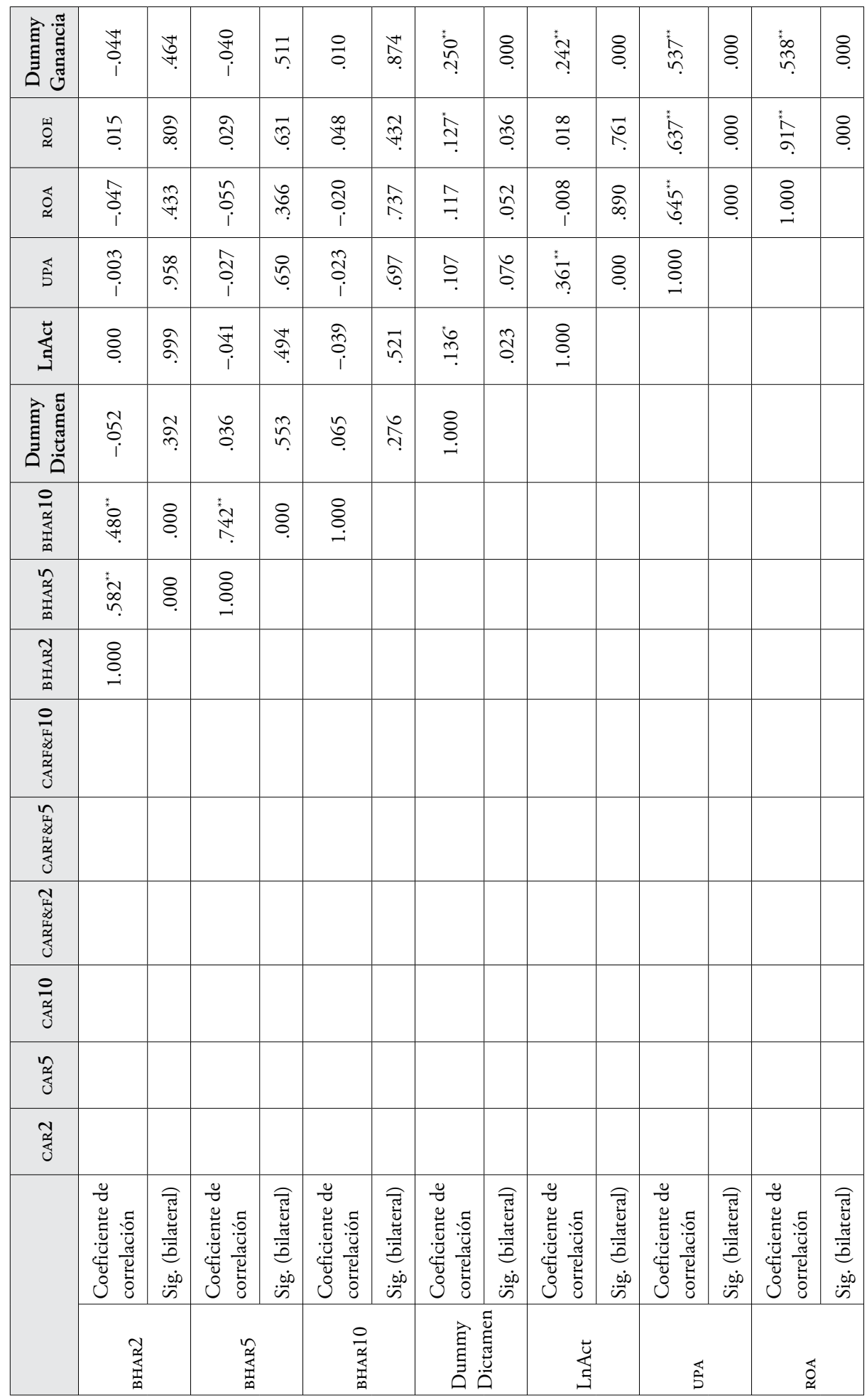




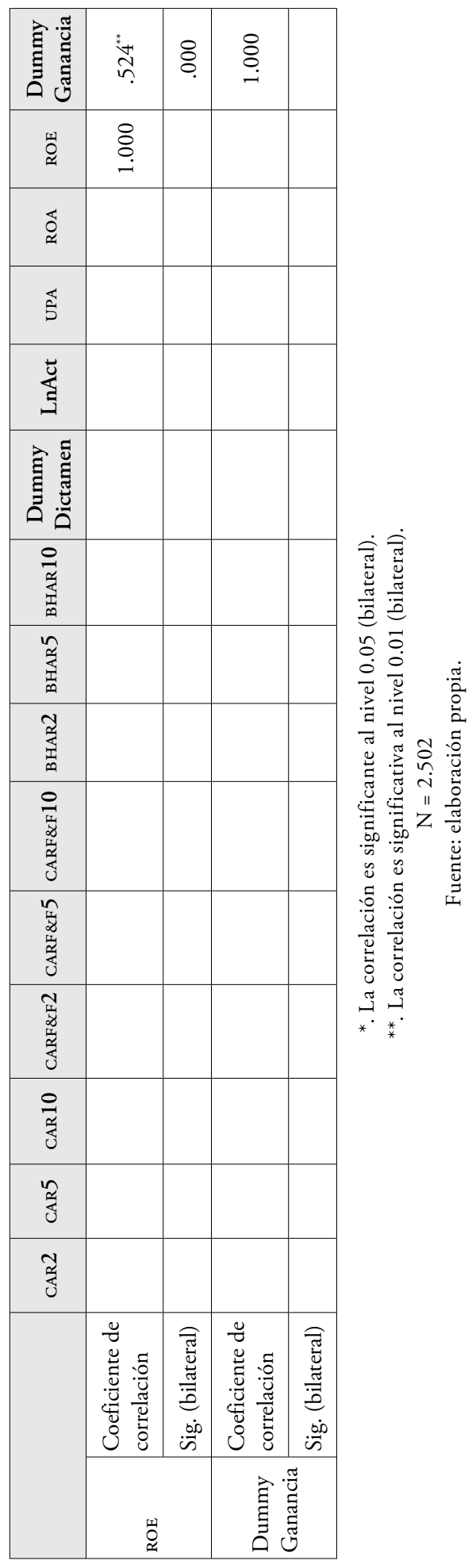




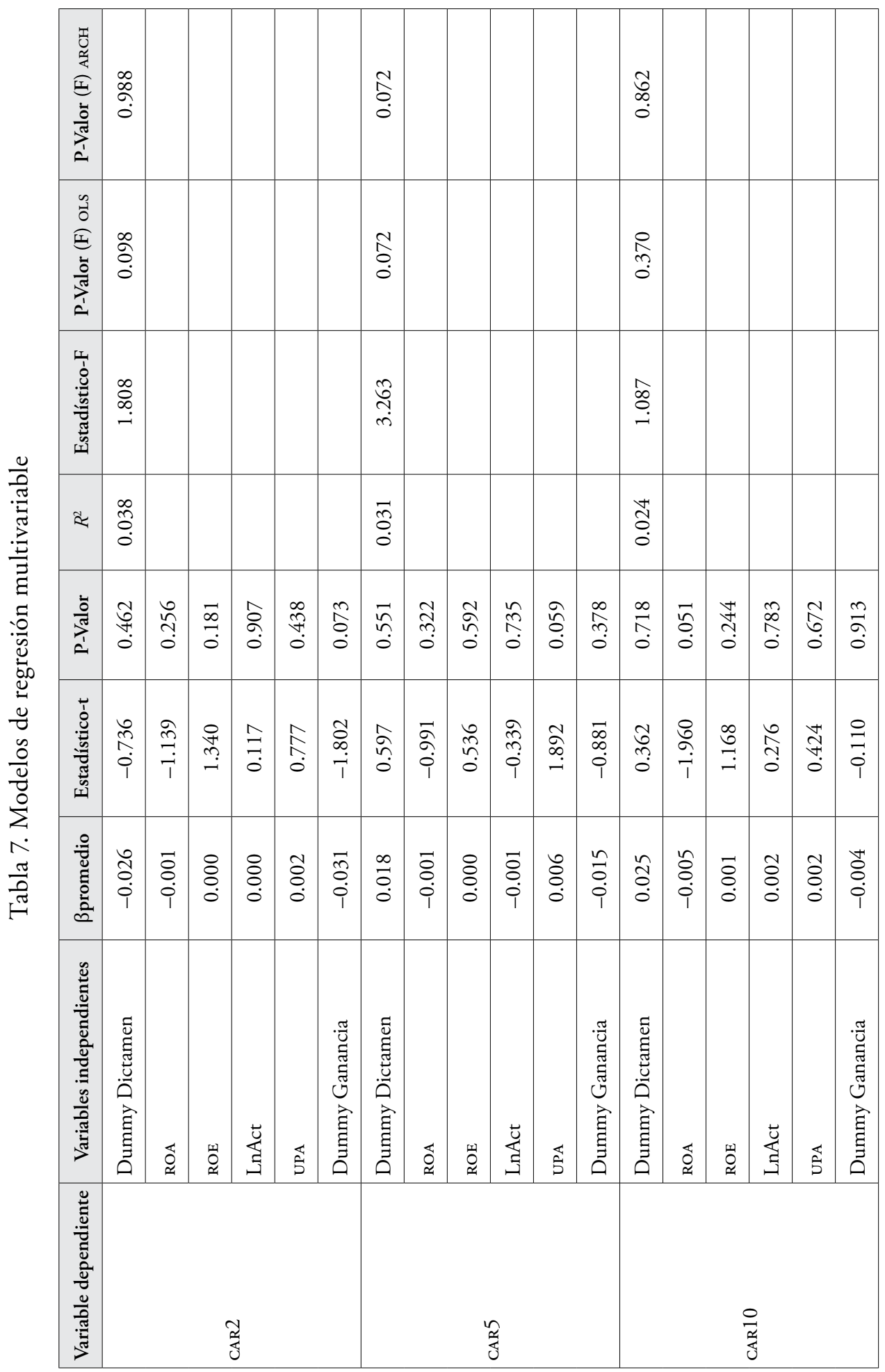




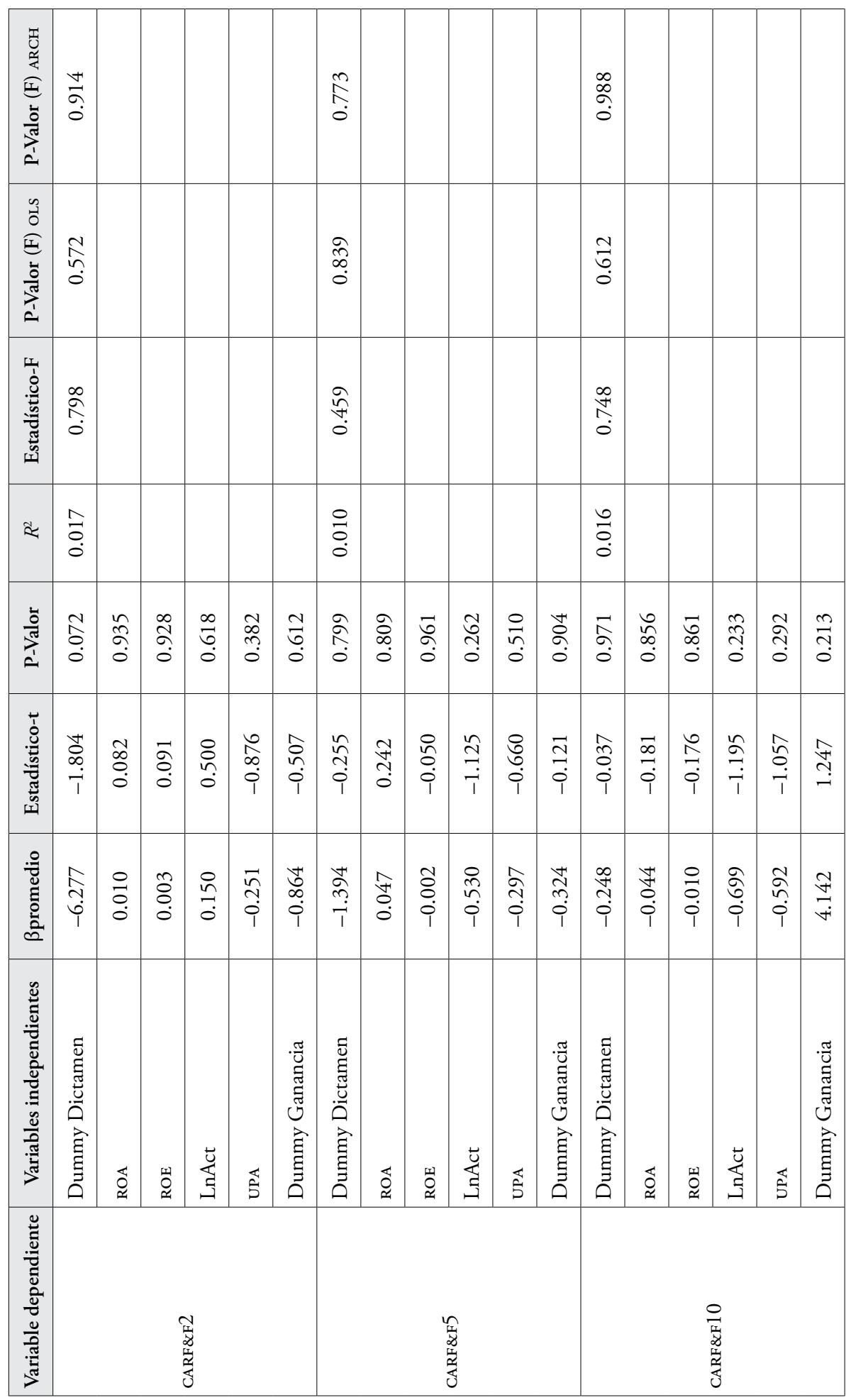




\begin{tabular}{|c|c|c|c|c|c|c|c|c|c|c|c|c|c|c|c|c|c|c|}
\hline 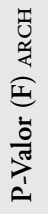 & $\begin{array}{l}\stackrel{+}{+} \\
\tilde{0}\end{array}$ & & & & & & $\stackrel{\grave{m}}{\stackrel{0}{0}}$ & & & & & & $\begin{array}{l}\stackrel{0}{n} \\
\tilde{n}\end{array}$ & & & & & \\
\hline 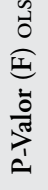 & $\begin{array}{l}\mathfrak{n} \\
\stackrel{0}{0} \\
0\end{array}$ & & & & & & 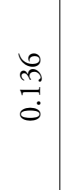 & & & & & & 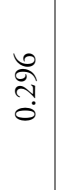 & & & & & \\
\hline 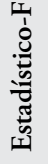 & $\underset{\sigma}{\rightleftarrows}$ & & & & & & 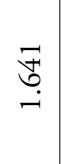 & & & & & & 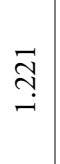 & & & & & \\
\hline$\approx$ & $\begin{array}{l}\exists \\
\stackrel{F}{0} \\
0\end{array}$ & & & & & & $\begin{array}{l}\hat{n} \\
\stackrel{0}{0}\end{array}$ & & & & & & $\begin{array}{l}\stackrel{0}{ } \\
\text { Oִ } \\
\stackrel{0}{0}\end{array}$ & & & & & \\
\hline 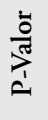 & $\begin{array}{l}\stackrel{0}{n} \\
\text { ñ } \\
0\end{array}$ & $\begin{array}{l}\infty \\
\exists \\
0\end{array}$ & $\begin{array}{l}\hat{\sigma} \\
\stackrel{0}{0}\end{array}$ & $\underset{\overbrace{}}{\stackrel{+}{\sigma}}$ & $\begin{array}{l}0 \\
\infty \\
\dddot{0} \\
\check{0}\end{array}$ & $\begin{array}{l}\cong \\
\Xi \\
\overrightarrow{0}\end{array}$ & $\begin{array}{l}\vec{n} \\
\overrightarrow{0}\end{array}$ & $\begin{array}{l}\tilde{\hat{n}} \\
\tilde{0}\end{array}$ & $\begin{array}{l}\stackrel{N}{+} \\
\stackrel{\infty}{0} \\
\stackrel{0}{0}\end{array}$ & 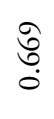 & $\begin{array}{l}\stackrel{+}{\infty} \\
\text { r. } \\
0\end{array}$ & $\stackrel{\vec{N}}{\overrightarrow{0}}$ & $\begin{array}{c}\stackrel{2}{ } \\
\stackrel{0}{0}\end{array}$ & 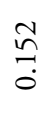 & $\stackrel{\hat{a}}{\grave{0}}$ & $\begin{array}{l}\tilde{\hat{n}} \\
\infty \\
0\end{array}$ & 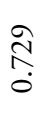 & \begin{tabular}{l}
$\infty$ \\
$\infty$ \\
\multirow{1}{0}{} \\
0
\end{tabular} \\
\hline 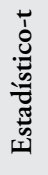 & $\begin{array}{l}\stackrel{0}{~} \\
\stackrel{\leftrightarrow}{i}\end{array}$ & \begin{tabular}{l}
$\widehat{O}$ \\
$\stackrel{\sim}{i}$ \\
\multirow{1}{*}{}
\end{tabular} & $\begin{array}{l}0 \\
\infty \\
\infty \\
\text { ஸे }\end{array}$ & $\begin{array}{l}\text { Tु } \\
\text { ִָ } \\
0\end{array}$ & \begin{tabular}{l} 
Dे \\
\multirow{+}{n}{} \\
$\tilde{o}$
\end{tabular} & $\begin{array}{l}\tilde{b} \\
\stackrel{\sim}{T}\end{array}$ & $\underset{\underset{+}{+}}{\stackrel{F}{+}}$ & $\begin{array}{l}\tilde{n} \\
\sigma \\
0 \\
i\end{array}$ & $\begin{array}{l}8 \\
\text { N̦ } \\
\stackrel{i}{1}\end{array}$ & $\begin{array}{l}\stackrel{\infty}{7} \\
\stackrel{+}{+} \\
\stackrel{1}{0}\end{array}$ & $\begin{array}{c}\mathbb{N} \\
\infty \\
0\end{array}$ & 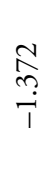 & $\begin{array}{l}\stackrel{\sim}{\tilde{n}} \\
\end{array}$ & 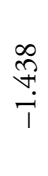 & $\stackrel{\overbrace ָ}{\overparen{I}}$ & 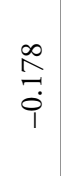 & $\underset{\stackrel{f}{f}}{\stackrel{0}{0}}$ & 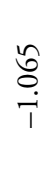 \\
\hline 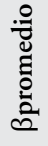 & 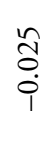 & 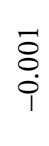 & $\begin{array}{l}8 \\
\stackrel{8}{\circ} \\
\dot{0}\end{array}$ & $\begin{array}{l}\overline{8} \\
\stackrel{0}{\circ}\end{array}$ & $\begin{array}{l}\overrightarrow{8} \\
\stackrel{0}{\circ}\end{array}$ & $\begin{array}{l}\text { ठิ } \\
0 \\
\stackrel{+}{1}\end{array}$ & $\begin{array}{l}\tilde{n} \\
\tilde{0} \\
0\end{array}$ & $\begin{array}{l}\vec{\circ} \\
\circ \\
0 \\
\end{array}$ & $\begin{array}{l}8 \\
\stackrel{8}{\circ} \\
\stackrel{0}{0}\end{array}$ & $\begin{array}{l}\overrightarrow{8} \\
\text { Oे } \\
\dot{\varphi}\end{array}$ & $\begin{array}{l}n \\
\stackrel{8}{0} \\
\stackrel{0}{0}\end{array}$ & $\begin{array}{l}\stackrel{H}{ } \\
\text { Ọ } \\
\stackrel{+}{1}\end{array}$ & $\begin{array}{c}\text { I } \\
0 \\
0 \\
0\end{array}$ & $\begin{array}{l}\text { Oे } \\
\text { } \\
\dot{\varphi}\end{array}$ & $\begin{array}{l}\square \\
\stackrel{\circ}{\circ}\end{array}$ & $\begin{array}{l}\overrightarrow{8} \\
\dot{\varphi} \\
\dot{1}\end{array}$ & $\begin{array}{l}\text { ㅇ. } \\
\stackrel{0}{0} \\
\stackrel{0}{0}\end{array}$ & $\begin{array}{l}\stackrel{0}{\Xi} \\
0 \\
\stackrel{0}{1}\end{array}$ \\
\hline 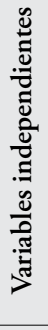 & 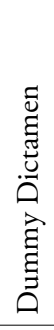 & $\begin{array}{l}\overleftarrow{\&} \\
\end{array}$ & $\stackrel{\text { II }}{\stackrel{2}{\approx}}$ & 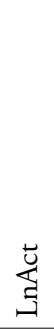 & $\overleftrightarrow{5}$ & 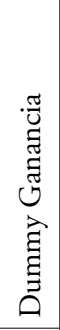 & 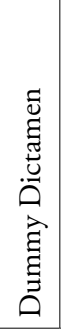 & 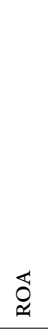 & $\begin{array}{l}\text { 띰 } \\
\stackrel{2}{*}\end{array}$ & 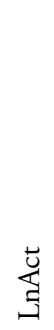 & $\overleftrightarrow{5}$ & 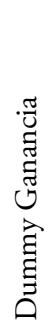 & 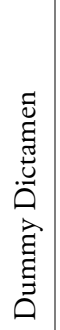 & $\underset{\mathscr{\Omega}}{\overleftarrow{\Omega}}$ & $\begin{array}{l}\text { rl } \\
\stackrel{2}{\pi}\end{array}$ & $\underset{J}{\breve{G}}$ & $\overleftrightarrow{\vdots}$ & 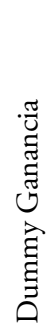 \\
\hline 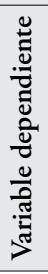 & & & & & & & & & & & & & & & & 总 & & \\
\hline
\end{tabular}


Tabla 8. Correlaciones por cópulas

\begin{tabular}{|c|c|c|c|c|c|c|c|c|c|c|}
\hline & CAR2 & CAR5 & CAR 10 & CARF\&F2 & CARF\& $\% 5$ & CARF $\& F 10$ & BHAR2 & BHAR 5 & BHAR 10 & $\begin{array}{l}\text { Dummy } \\
\text { Dictamen }\end{array}$ \\
\hline CAR2 & 1 & 0.64 & 0.48 & 0.33 & 0.25 & 0.14 & 0.85 & 0.53 & 0.43 & -0.25 \\
\hline CAR5 & & 1 & 0.72 & 0.18 & 0.31 & 0.22 & 0.49 & 0.82 & 0.59 & 0.02 \\
\hline CAR 10 & & & 1 & 0.05 & 0.23 & 0.26 & 0.35 & 0.64 & 0.79 & -0.08 \\
\hline CARF\&F2 & & & & 1 & 0.63 & 0.41 & 0.20 & 0.13 & 0.03 & -0.31 \\
\hline CARF\&F 5 & & & & & 1 & 0.76 & 0.15 & 0.24 & 0.20 & -0.17 \\
\hline CARF $\& F 10$ & & & & & & 1 & 0.09 & 0.22 & 0.25 & -0.04 \\
\hline BHAR2 & & & & & & & 1 & 0.61 & 0.52 & -0.22 \\
\hline BHAR 5 & & & & & & & & 1 & 0.78 & 0.11 \\
\hline BHAR 10 & & & & & & & & & 1 & 0.21 \\
\hline $\begin{array}{l}\text { Dummy } \\
\text { Dictamen }\end{array}$ & & & & & & & & & & 1 \\
\hline
\end{tabular}

Fuente: elaboración propia.

Ahora bien, con el fin de en robustecer los resultados iniciales de que el dictamen del auditor tiene un impacto sobre los activos del mercado de capitales del MILA, se presenta gráficamente las cópulas de la correlación de los retornos anormales acumulados con la variable dictamen (ver gráfico 4).

Gráfico 4. Cópulas de correlación gaussianas entre series de retornos anormales acumulados y dictamen en el MILA

CAR 2

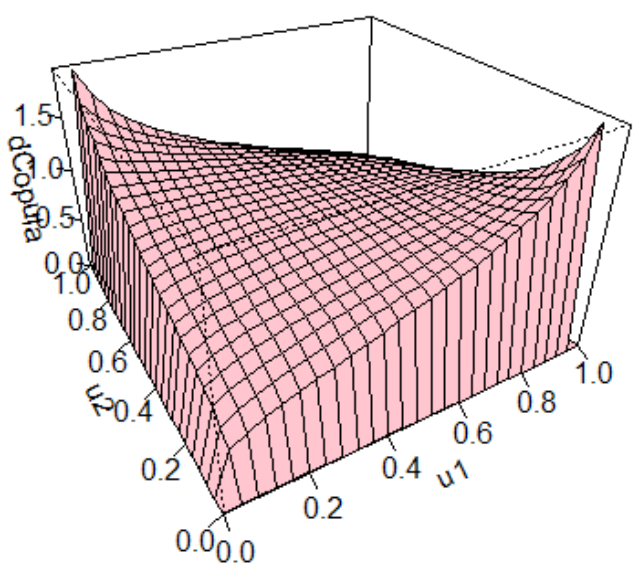


CAR5

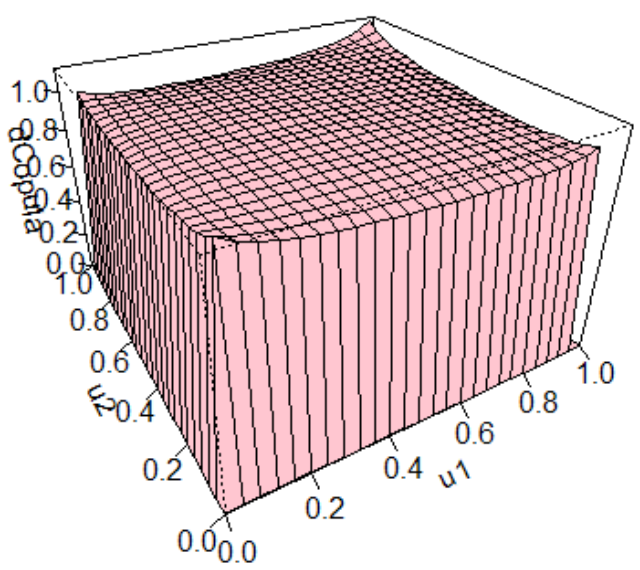

CAR 10

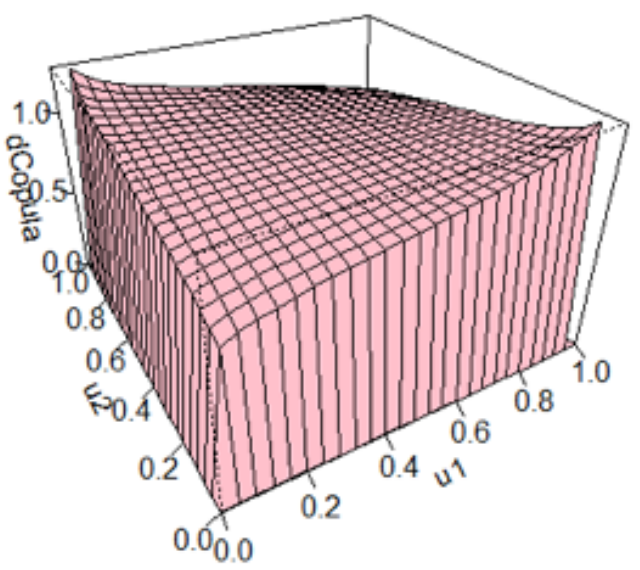

CARF\&F2

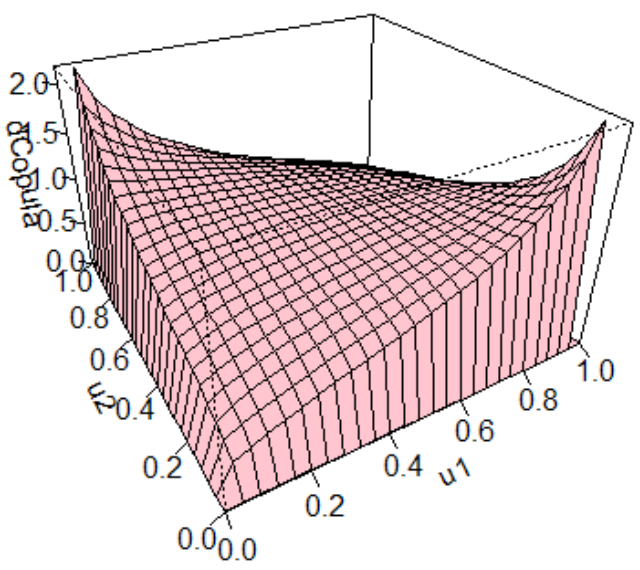




\section{CARF\&F 5}

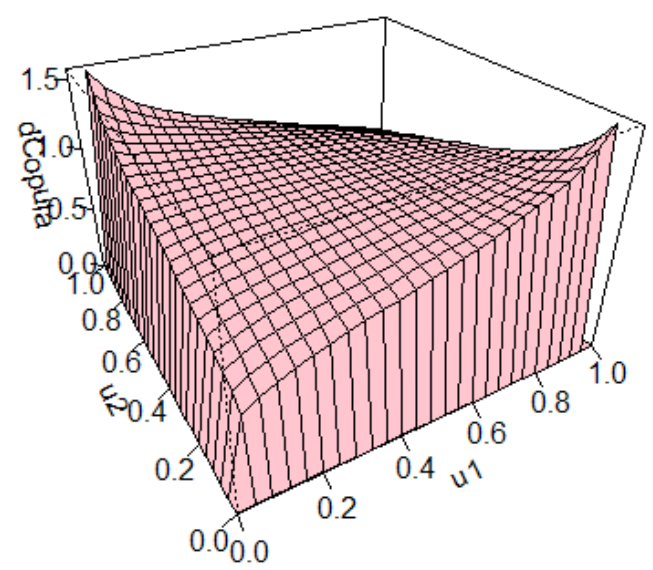

CARF \&F10

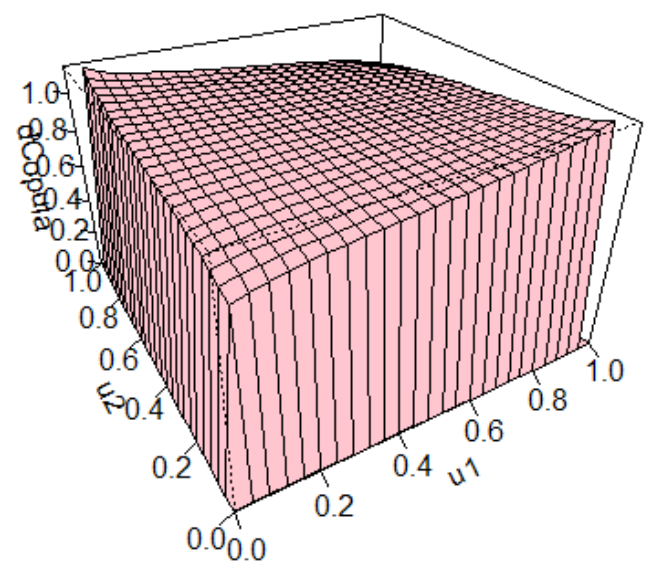

BHAR2

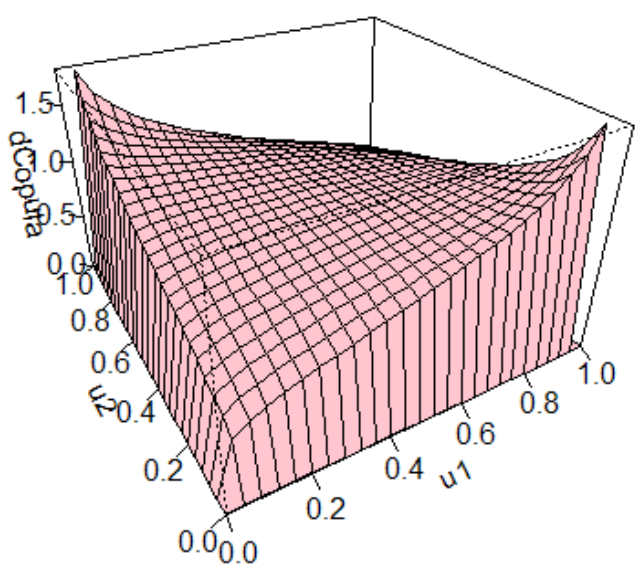




\section{BHAR5}

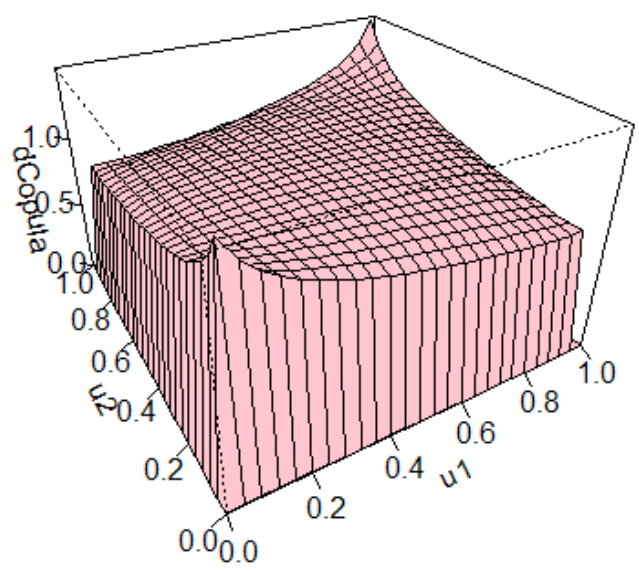

BHAR 10

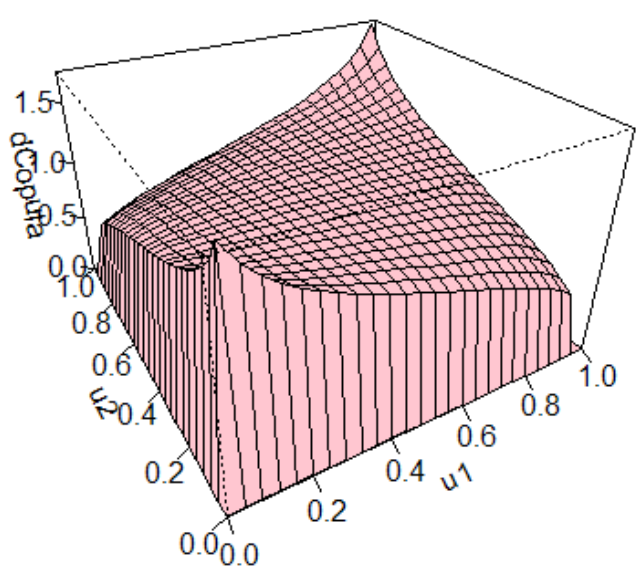

Fuente: elaboración propia.

En el gráfico 4 se ilustra dada una de las densidades de las cópulas de las correlaciones estimadas, en ellas se puede identificar que los gráficos son asimétricos y no convergen a la cópula gaussiana, por tanto, se puede concluir empíricamente por medio de las cópulas multivariantes que no hay una correlación significativa entre el dictamen y los retornos anormales acumulados entre los ańos de estudio analizados en la investigación.

\section{Conclusiones}

Esta investigación se llevó a cabo utilizando la metodología de estudios de eventos, buscando evaluar el impacto generado por la divulgación de los informes de auditoría de las empresas que cotizan en el mercado integrado latinoamericano (MILA), en el periodo 2011 al 2017, pretendiendo medir si existen cambios significativos en los retornos de las acciones cotizadas durante este lapso y, por tanto, poder verificar si 
el trabajo de los auditores influye en la volatilidad de los precios de los activos de este mercado integrado. Como lo ilustrado en la tabla 3, se verifica que gran parte de las acciones de la muestra tienen retornos anormales significativos, el cual va aumentando con el tamaño de la ventana de evento sin importar el modelo planteado para el cálculo del retorno anormal, esto indica que, aunque los dictámenes de auditoria impactan sobre algunas empresas de forma significativa, no se puede afirmar que los dictámenes influyan sobre toda la muestra, como se observa en las tablas de 6 y 8 donde realizó el análisis correlacional entre las diferentes variables CAR estimadas y la variable utilizada como representante de los dictámenes de auditoria, los cuales, en general, no son significativos.

Este estudio brinda evidencia empírica para afirmar que los inversionistas del mercado integrado mILA no deben utilizar los informes de auditoría, como un factor determinante en la toma de decisiones para participar activamente en este mercado, dado que en este estudio con diferentes análisis estadísticos se verificó que hoy existe un aporte significativo en la volatilidad de las acciones del mercado. Por último, igualmente podemos indicar que, a pesar de que se utilizan variables que hacen parte de los estados financieros de las empresas, las cuales según la literatura nos pueden indicar un factor importante de las empresas, como es la continuidad, esta información no interfiere en gran escala en la toma de decisiones de los inversionistas, aclarando que para estudios futuros se podría considerar incorporar en el modelo factores macroeconómicos.

\section{REFERENCIAS BIBLIOGRÁFICAS}

Akgi, V. (1989). Conditional heteroscedasticity in time series of stock returns: Evidence and forecasts. Journal of Business, 62(1), 55-80.

Barber, B. y Lyon, J. (1997). Detecting long-run abnormal stock returns: The empirical power and specification of test statistics. Journal of Financial Economics, (43), 341372. doi: https://doi.org/10.1016/S0304-405X(96)00890-2

Bollerslev, T. (1986). Generalized autoregressive conditional heteroscedasticity. Journal of Econometrics, 31(3), 307-327.

Cardona, D. (2012). Dependencia estructural en los mercados bursátiles de Colombia y Estados Unidos: una aproximación usando cópulas. Cuadernos de Economía, 31, 147-178.

Chen, K. y Church, B. (1996). Going concern opinions and the market's reaction to bankruptcy filings. The Accounting Review, 71(1), 117-128.

Chiang, T. y Doong, S. (2001). Empirical analysis of stock returns and volatility: Evidence from seven Asian stock markets based on TAR-GARCH Model. Review of Quantitative Finance and Accounting, 17(3), 301-318.

Corbo, V. (1997). Integración financiera en América latina. Documentos de trabajo de la oficina de economista jefe. Banco Interamericano de Desarrollo, 1-26. 
Dopuch, N., Houlthausen, R. y Leftwich, R. (1986). Abnormal stock returns associated with media disclosures of "subject to" qualified audit opinions. Journal of Accounting and Economics, 8(2), 93-117. doi: https://doi.org/10.1016/0165-4101(86)90013-3

Dye, R. (1993). Auditor status, legal liability, and auditor wealth. Journal of Political Economy, 101(5), 887-914.

Fama, E. (1991). Efficient capital markets II. The Journal of Finance, 46(5), 1575-1617.

Fama, E. y French, K. (1993). Common risk factors in the returns on stocks and bonds. Journal of Financial Economics, 33(1), 3-56. doi: http://doi.org/10.1016/0304-405X(93)90023-5

Fama, E. y Laffer, A. (1971). A Information and capital markets. Journal of Business, 44(3), 289-298.

Franco, H. y Marra, E. (2000). Auditoria contábil (3 ed.). São Paulo: Atlas.

$\mathrm{Fu}, \mathrm{F}$. (2009). Idiosyncratic risk and the cross-section of expected stock returns. Journal of Financial Economics, 91(1), 24-37. doi: http://doi.org/10.1016/j.jfineco.2008.02.003

López Domínguez, I. (2007). El proceso de integración de los mercados financieros en Europa. Revista Escuela de Administración de Negocios, (59), 87-97.

Mackinlay, A. C. (1997). Event studies in economics and finance. Journal of Economic Literature, 35, 13-39.

Martínez, M., Martínez, A. y Benau, M. (2004). Reaction of the Spanish capital market to qualified audit reports. European Accounting Review, 13(4), 93-117. doi: https:// doi.org/10.1080/0963818042000216848

Mendonça, F., Klotzle, M., Pinto, A. y Montezano, R. (2012). A relação entre risco idiossincrático e retorno no mercado acionário brasileiro. Revista Contabilidade \& Finanças, 23(60), 246-257.

Mendoza, A. y Galvanovskis, E. (2014). La cópula ged bivariada:una aplicación en entornos de crisis. El trimestre económico, 81(323), 721-746. doi: http://dx.doi.org/10.20430/ ete.v81i323.128

Nieto, B. (2001). Los modelos multifactoriales de valoración de activos: Un análisis empírico comparativo. IVIE Working Paper, 29(w P-EC 2001-19).

Pei, D., Opong, K. y Hamill, P. (2004). An analysis of the information content of qualified audit reports: Evidence from China's Shenzhen Market. Working Paper.

Romero, Y., Ramírez, F. y Guzmán, D. (2013). Mercado Integrado Latinoamericanos (MILA): análisis de correlación y diversificación de los portafolios de acciones de los tres países miembros en el periodo 2007-2012. Cuadernos de Contabilidad, $14(34), 53-74$.

Soltani, B. (2000). Some empirical evidence to support the relationship between audit reports and stock prices- The French case. International Journal of Auditing, 4(3), 269-291. doi: https://doi.org/10.1111/1099-1123.00317

Vargas, W. y Bayardo, J. (2013). El mila. Mercado de integración entre Chile, Perú y Colombia. Revista de Relaciones Internacionales, Estrategia y Seguridad, 1(8), 113133. doi: https://doi.org/10.18359/ries.67

Wallace, W. (1987). The economic role of the audit in free and regulated markets: a review. Research in Accounting Regulation, 1, 7-34. 